\title{
Association of genetic variation in Wnt signaling pathway gene ( $\beta$-catenin) with susceptibility of Leukoaraiosis
}

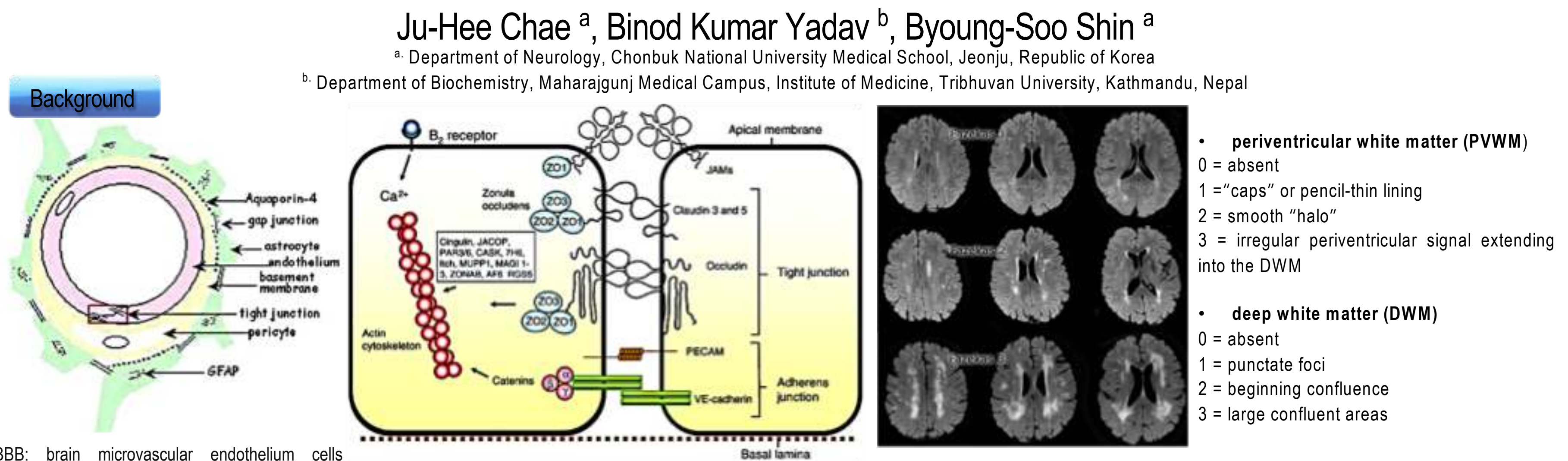

BBB: brain microvascular endothelium cells (BMVEC), astrocytes, basement membrane, and pericytes and neuron

the role of wnt/ $\beta$-catenin pathway as a key regulator of the $B B B$ and it seems, therefore, a good candidate gene for LA.

\section{Method}

- Study population

Total of 339 LA cases (LA-PVWM, 183; LA-DWM 156) vs controls (LA-PVWM, 203; LA-DWM 230)

- Genotyping

Three single-nucleotide polymorphisms (SNPs), rs1880481C>A (SNP1), rs13072632 C>T (SNP2) and rs4135385 A>G (SNP3), were genotyped by RT-PCR with LightCycler 2.0 RT-PCR (Roche, Germany).

\section{Results}

Table 1. Demographic and clinical characteristics in control and patients with $L A$

\begin{tabular}{|c|c|c|c|c|c|c|}
\hline Characteristics & Control $(n=203)$ & $\begin{array}{c}\text { LA-PVWM ( } \mathrm{n}= \\
183)\end{array}$ & $p$ & Control $(n=230)$ & $\begin{array}{c}\text { LA-DWM } \\
(n=156)\end{array}$ & $p$ \\
\hline Age & $61.66 \pm 13.01$ & $74.46 \pm 7.76$ & $<0.001$ & $63.86 \pm 13.25$ & $73.44 \pm 8.87$ & $<0.001$ \\
\hline Gender (M), n (\%) & 127 (62.6\%) & $91(49.7 \%)$ & 0.11 & $143(62.2 \%)$ & $75(48.1 \%)$ & 0.006 \\
\hline $\begin{array}{c}\text { Hypertension, } \\
\mathrm{n}(\%)\end{array}$ & $112(55.4 \%)$ & $134(73.2 \%)$ & $<0.001$ & $133(58.1 \%)$ & $113(72.4 \%)$ & 0.004 \\
\hline Diabetes, n (\%) & $58(28.7 \%)$ & 65 (35.5\%) & 0.153 & $71(31.0 \%)$ & $52(33.3 \%)$ & 0.63 \\
\hline Cholesterol (mg\%) & $166.13 \pm 43.51$ & $169.21 \pm 41.13$ & 0.477 & $166.66 \pm 43.15$ & $168.97 \pm 41.25$ & 0.62 \\
\hline Triglyceride (mg\%) & $\begin{array}{c}147.12 \pm \\
130.59\end{array}$ & $134.62 \pm 104.08$ & 0.201 & $\begin{array}{c}148.80 \pm \\
130.52\end{array}$ & $126.46 \pm 98.13$ & 0.07 \\
\hline HDL-Chol (mg\%) & $40.40 \pm 10.88$ & $41.26 \pm 12.02$ & 0.459 & $40.43 \pm 10.75$ & $41.36 \pm 12.37$ & 0.43 \\
\hline LDL-Chol (mg\%) & $98.43 \pm 34.89$ & $97.92 \pm 31.90$ & 0.881 & $98.08 \pm 34.65$ & $98.36 \pm 31.47$ & 0.93 \\
\hline Chol/HDL & $4.28 \pm 1.21$ & $4.31 \pm 1.28$ & 0.802 & $4.29 \pm 1.23$ & $4.30 \pm 1.26$ & 0.96 \\
\hline LDL/HDL & $2.54 \pm 0.95$ & $2.51 \pm 0.93$ & 0.747 & $2.53 \pm 0.95$ & $2.51 \pm 0.93$ & 0.89 \\
\hline ApoA1 (mg\%) & $1.23 \pm 0.78$ & $1.19 \pm 0.26$ & 0.56 & $1.21 \pm 0.72$ & $1.20 \pm 0.27$ & 0.81 \\
\hline $\mathrm{ApoB}(\mathrm{mg} \%)$ & $0.97 \pm 0.29$ & $0.93 \pm 0.25$ & 0.301 & $0.96 \pm 0.28$ & $0.92 \pm 0.26$ & 0.23 \\
\hline ApoA1/ApoB & $0.84 \pm 0.31$ & $0.81 \pm 0.27$ & 0.367 & $0.85 \pm 0.30$ & $0.79 \pm 0.27$ & 0.06 \\
\hline Lp(a) (mg\%) & $30.42 \pm 26.09$ & $28.23 \pm 26.13$ & 0.46 & $31.12 \pm 28.39$ & $26.65 \pm 21.86$ & 0.14 \\
\hline Glucose (mg\%) & $144.80 \pm 65.91$ & $141.78 \pm 81.78$ & 0.693 & $145.28 \pm 65.27$ & $140.51 \pm 85.08$ & 0.54 \\
\hline $\mathrm{HbA1C}(\%)$ & $6.36 \pm 3.53$ & $6.21 \pm 1.38$ & 0.595 & $6.35 \pm 3.34$ & $6.19 \pm 1.40$ & 0.58 \\
\hline $\mathrm{tHCy}(\mu \mathrm{mol} / \mathrm{L})$ & $13.26 \pm 6.30$ & $14.34 \pm 5.78$ & 0.081 & $13.29 \pm 6.30$ & $14.48 \pm 5.67$ & 0.06 \\
\hline
\end{tabular}

These findings suggest that advanced age and hypertension are the aggravating factor for the LA and can influence the risk estimation of genetic factors in LA.

Table 4. Haplotype frequency of $\beta$-catenin in control an LA

\begin{tabular}{ccccc}
\hline Genotype & Control & LA-PVWM & OR $(95 \% \mathrm{Cl})$ & $\mathrm{p}$-value \\
\hline SNP1/SNP2/SNP3 & & & & \\
C-C-A & 0.0059 & 0.0048 & $1.11(0.05-7.92)$ & 0.91 \\
A-C-A & 0.1242 & 0.201 & $0.9(0.63-1.27)$ & 0.33 \\
C-T-A & 0.493 & 0.2914 & $\mathbf{0 . 4 2}(0.31-0.57)$ & $<0.0001$ \\
A-T-A & 0 & 0.0465 & $\mathbf{4 0 . 7}(2.43-679.89)$ & $<0.0001$ \\
C-C-G & 0.0065 & 0.0041 & $0.156(0.019-1.276)$ & 0.05 \\
A-C-G & 0.0826 & 0.0251 & $\mathbf{0 . 2 8 5}(0.134-0.60)$ & $\mathbf{0 . 0 0 0 5}$ \\
C-T-G & 0.2828 & 0.4019 & $0.108(0.06-0.188)$ & $<0.0001$ \\
A-T-G & 0.005 & 0.0252 & $\mathbf{5 . 0 9}(1.09-23.73)$ & $\mathbf{0 . 0 2 1}$ \\
& & & & \\
\hline Genotype & Control & LA-DWM & OR(95\%Cl) & p-value \\
\hline SNP1/SNP2/SNP3 & & & & \\
C-C-A & 0.0058 & 0.0035 & $0.5(0.05-4.73)$ & 0.52 \\
A-C-A & 0.1296 & 0.2141 & $1.82(1.24-2.67)$ & 0.0019 \\
C-T-A & 0.4558 & 0.3004 & $\mathbf{0 . 5 1}(0.37-0.69)$ & $<0.0001$ \\
A-T-A & 0.0066 & 0.0494 & $\mathbf{7 . 6 9}(2.20-26.81)$ & $\mathbf{0 . 0 0 0 2}$ \\
C-C-G & 0.0073 & 0.0036 & $0.5(0.05-4.73)$ & 0.52 \\
A-C-G & 0.066 & 0.0321 & $\mathbf{0 . 4 7}(0.22-0.98)$ & $\mathbf{0 . 0 4}$ \\
C-T-G & 0.3289 & 0.3657 & $1.17(0.87-1.59)$ & 0.28 \\
A-T-G & 0 & 0.314 & $\mathbf{3 1 . 9 7}(1.86-548.0)$ & $\mathbf{0 . 0 0 0 1}$ \\
\hline
\end{tabular}

Table 2a. Comparison of genetic frequency of $\beta$-catenin polymorphisms in the control and LA-PVWM $\begin{array}{ccccc}\text { Genotypes } & \begin{array}{c}\text { Control } \\ (n=203 \%)\end{array} & \begin{array}{c}\text { LA-PVWM } \\ (n=183 \%)\end{array} \quad \text { OR }(95 \% \mathrm{Cl}), p & \text { AOR }(95 \% \mathrm{Cl}), p\end{array}$

\begin{tabular}{ccccc}
\hline SNP1 & & & & \\
CC & $125(61.6)$ & $93(50.8)$ & 1.0 (Reference) & 1.0 (Reference) \\
AC & $70(34.5)$ & $71(38.8)$ & $1.36(0.89-2.086), 0.15$ & $1.14(0.69-1.89), 0.58$ \\
AA & $8(3.9)$ & $19(10.4)$ & $\mathbf{3 . 1 9}(\mathbf{1 . 3 3 - 7 . 6 1 ) , 0 . 0 0 6}$ & $2.37(0.82-6.84), 0.108$ \\
Dominant & & & & \\
CC vs AC+AA & $125 / 78$ & $93 / 80$ & $\mathbf{1 . 5 5}(\mathbf{1 . 0 3 - 2 . 3 2}), \mathbf{0 . 0 3 3}$ & $1.28(0.79-2.07), 0.30$ \\
Recessive & & & & \\
CC+AC vs AA & $175 / 8$ & $167 / 19$ & $\mathbf{2 . 4 8 ( 1 . 0 6 1 - 5 . 8 4 ) , 0 . 0 3 1 2}$ & $2.35(0.84-6.53), 0.105$
\end{tabular}

\begin{tabular}{|c|c|c|c|c|}
\hline \multicolumn{5}{|l|}{ SNP3 } \\
\hline AA & $83(40.9)$ & $51(27.9)$ & 1.0 (Reference) & 1.0 (Reference) \\
\hline$A G$ & $87(42.9)$ & $97(53.0)$ & $1.81(1.15-2.85), 0.0097$ & $1.87(1.81-3.23), 0.022$ \\
\hline GG & $33(16.3)$ & $35(19.1)$ & $1.76(0.95-3.113), 0.068$ & $1.62(0.80-3.27), 0.17$ \\
\hline \multicolumn{5}{|l|}{ Dominant } \\
\hline $\begin{array}{l}\text { AA vs } \mathbf{A G + G G} \\
\text { Recessive }\end{array}$ & $83 / 120$ & $51 / 132$ & $1.79(1.16-2.74), 0.0073$ & $1.79(1.07-2.98), 0.0247$ \\
\hline$A A+A G$ vs $G G$ & $170 / 33$ & $148 / 35$ & $1.21(0.72-2.05), 0.45$ & $1.12(0.60-2.10), 0.70$ \\
\hline \multicolumn{5}{|c|}{ Table $2 \mathrm{~b}$. Comparison of genetic frequency of $\beta$-catenin polymorphisms in the control and LA-DWM } \\
\hline Genotypes & $\begin{array}{c}\text { Control } \\
(n=230, \%)\end{array}$ & $\begin{array}{l}\text { LA-DWM } \\
(n=156, \%)\end{array}$ & OR (95\%Cl), p & $\operatorname{AOR}(95 \% \mathrm{Cl}), \mathrm{p}$ \\
\hline \multicolumn{5}{|l|}{ SNP1 } \\
\hline $\mathrm{CC}$ & $146(63.5)$ & $72(46.2)$ & 1.0(Reference) & 1.0 (Reference) \\
\hline$A C$ & $75(32.6)$ & $66(42.3)$ & $1.78(1.15-2.75), 0.008$ & $1.64(1.02-2.63), 0.039$ \\
\hline $\begin{array}{c}\text { AA } \\
\text { Dominant }\end{array}$ & $9(3.9)$ & $18(11.5)$ & $4.05(1.73-9.47), 0.0006$ & $3.31(1.32-8.32), 0.01$ \\
\hline $\begin{array}{c}\text { CC vs AC+AA } \\
\text { Recessive }\end{array}$ & $146 / 84$ & $72 / 84$ & $2.02(1.34-3.067), 0.0008$ & $1.83(1.17-2.88), 0.008$ \\
\hline$C C+A C$ vs $A A$ & $221 / 9$ & $138 / 18$ & $3.20(1.39-7.32), 0.0039$ & $2.72(1.10-6.71), 0.029$ \\
\hline
\end{tabular}

$\begin{array}{lllll}\text { SNP3 } & & & \\ \text { AA } & 92(40.0) & 42(26.9) & 1.0 \text { (Reference) } & 1.0 \text { (Reference) }\end{array}$

AG $\quad 91(39.6) \quad 93(59.6) \quad 2.23(1.40-3.56), 0.0006 \quad 2.34(1.38-3.95), 0.0014$

GG $\quad 47(20.4) \quad 21(13.5) \quad 0.97(0.52-1.83), 0.94 \quad 0.82(0.41-1.65), 0.59$

Dominant

AA vs AG+GG $\quad 92 / 138 \quad 42 / 114 \quad 1.81(1.16-2.81), 0.008 \quad 1.79(1.10-2.91), 0.018$

Recessive

$\begin{array}{lllll}\text { AA+AG vs GG } \quad 183 / 47 & 135 / 21 & 0.60(0.34-1.06), 0.077 & 0.50(0.27-0.93), 0.02\end{array}$

- The genotype distribution of SNP1 and SNP3 were significant different among both LA and control.

AOR: from logistic regression analysis with respect to age, sex, and hypertension.

Table 3. Combinatorial effects in LA and control according to the $\beta$-catenin genetic polymorphism

\begin{tabular}{ccccc}
\hline Genotype & Control & LA-PVWM & OR $(95 \% \mathrm{Cl})$ & $\mathrm{p}$ \\
\hline SNP1/SNP3 & & & & \\
CC/AA & 53 & 16 & & \\
AC/AG & 35 & 43 & $4.0(1.99-8.3)$ & $<0.0001$ \\
AC/GG & 9 & 6 & $2.2(0.68-7.15)$ & 0.17 \\
AA/AG & 1 & 4 & $13.25(1.38-127.2)$ & 0.0057 \\
AA/GG & 3 & 2 & $2.20(0.33-14.4)$ & 0.39 \\
(AC+AA)/(AG+GG) & 48 & 55 & $\mathbf{3 . 7 9}(1.92-7.49)$ & $<0.0001$ \\
& & & & \\
\hline Genotype & Control & LA-DWM & OR (95\%Cl) & $\mathrm{p}$ \\
\hline SNP1/SNP3 & & & & \\
CC/AA & 57 & 12 & & \\
AC/AG & 36 & 42 & $\mathbf{5 . 5 4}(2.57-11.91)$ & $<0.0001$ \\
AC/GG & 9 & 6 & $3.16(0.99-10.58)$ & 0.053 \\
AA/AG & 1 & 4 & $19.0(1.94-185.5)$ & 0.001 \\
AA/GG & 3 & 2 & $3.16(0.47-21.06)$ & 0.21 \\
(AC+AA)/(AG+GG) & 49 & 54 & $\mathbf{5 . 2 3}(2.51-10.90)$ & $<0.0001$ \\
\hline
\end{tabular}

Copyright @ 2017 J-H. CHAE, B.K. YADAV, B.-S. SHIN sbsoo@jbnu.ac.kr 